\title{
Circumstellar Environments of MYSOs Revealed by IFU Spectroscopy
}

\author{
F. Navarete ${ }^{1}$, A. Damineli ${ }^{1}$, C. L. Barbosa ${ }^{2}$ and R. D. Blum ${ }^{3}$ \\ ${ }^{1}$ IAG-USP, Rua do Matão, 1226, 05508-900, São Paulo, SP Brazil \\ email: navarete@usp.br \\ ${ }^{2}$ UNIVAP, São José dos Campos, SP, Brazil \\ ${ }^{3}$ NOAO, 950 N Cherry Ave., Tuczon, AZ 85719 USA
}

\begin{abstract}
Formation of massive stars $\left(M>8 M_{\odot}\right)$ is still not well understood and lacks of observational constraints. We observed 7 MYSO candidates using the NIFS spectrometer at Gemini North Telescope to study the accretion process at high angular resolution ( $\sim 50$ mas) and very closer to the central star. Preliminary results for 2 sources have revealed circumstellar structures traced by Brackett-Gamma, $\mathrm{CO}$ lines and extended $\mathrm{H}_{2}$ emission. Both sources present kinematics in the $\mathrm{CO}$ absorption lines, suggesting rotating structures. The next step will derive the central mass of each source by applying a keplerian model for these $\mathrm{CO}$ features.
\end{abstract}

Keywords. Stars: formation, Stars: early-type, Stars: pre-main sequence, Stars: circumstellar matter, Techniques: spectroscopic, Techniques: high angular resolution

\section{Introduction}

The formation mechanisms of massive stars is one of the most important problems in stellar astrophysics and still poorly understood. While low mass young stellar objects (YSOs) reach the zero age main sequence (ZAMS) after the accretion period is finished, the massive ones (MYSOs) reach the main sequence while the accretion is still ongoing.

Recently, Navarete et al. (2014) carried a survey of extended $\mathrm{H}_{2}$ emission towards a well defined sample of MYSO candidates. They found that $\sim 20 \%$ of the sample is associated to jet-like structures, suggesting bipolar outflows. These observational evidences supports the accretion scenario (Krumholz et al. 2005) and also indicate that massive stars cannot be merely formed by merging of low mass stars (Bonnell et al. 2001). Although the scenario of an accretion disk may apply for all massive stars, the details are lacking. There are a few examples of well documented accretion disks around massive $\left(10-20 \mathrm{M}_{\odot}\right)$ forming stars (Davies et al. 2010; Murakawa et al. 2013). Disks have been also identified through profile fitting of emission features seen in high spectral resolution data (Bik \& Thi 2004; Blum et al. 2004). In order to study the details of the circumstellar environment around MYSOs, we present K-band integral field spectroscopy of six MYSOs associated to large scale $\mathrm{H}_{2}$ outflows, identified by Navarete et al. (2014); and one source selected from Varricatt et al. (2010).

\section{Observations}

$K$-band $(\lambda \approx 2.2 \mu \mathrm{m}, \mathrm{R} \approx 5200)$ spectra of 7 MYSO candidates were obtained with the Near-infrared Integral Field Spectrograph (NIFS) deployed at the 8-m Gemini North Telescope (Hawaii). 

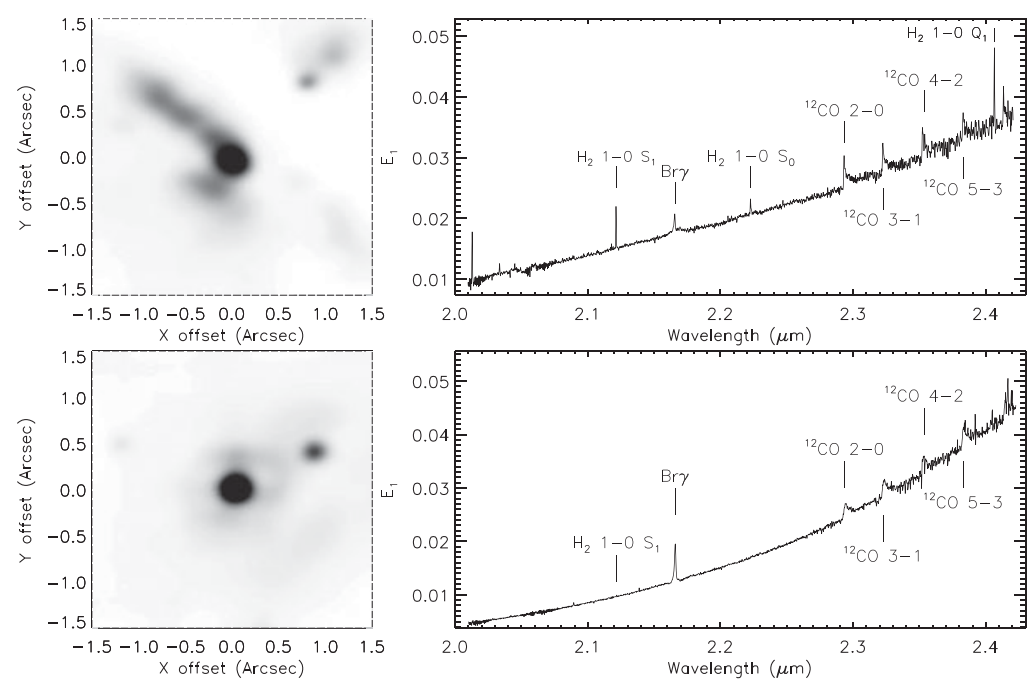

Figure 1. PCA results for two sources. Each component consists in a tomogram (left image) and an eigenspectrum (right plot). Upper panel: First component of the PCA analysis for source \#58. Lower Panel: First component for source \#A49.

Data reduction was performed using the same methodology described in Menezes et al. (2014) and the procedure for applying the PCA analysis on the datacubes are described in Steiner et al. (2009).

\subsection{Results}

Figure 1 presents the tomogram (left) and eigenspectrum (right) for the first component of \#58 source (upper panel), and components 1 and 4 of \#A49 source (middle and bottom panels). The first component of the PCA results basically displays the stellar spectrum, which corresponds to $\approx 90-99 \%$ of the datacube variance. The source \#58 exhibits a series of $\mathrm{H}_{2}$ transitions, a relatively broad $\mathrm{Br} \gamma$ feature and $\mathrm{CO}$ bandhead, all identified in emission. The eigenvector associated to the first component of the \#A49 source (middle panel) exhibits emission of both $\mathrm{Br} \gamma$ and $\mathrm{CO}$ bandhead. Several absorption features were identified at $\sim 2.4 \mu \mathrm{m}$.

\subsection{Summary and Conclusions}

The K-band IFU spectroscopy of \#58 and \#A49 sources revealed that: $i$ ) The circumstellar environment of MYSOs are mainly traced by $\mathrm{Br}-\gamma, \mathrm{H}_{2}$ and $\mathrm{CO}$ bandhead emission (and $\mathrm{CO}$ absorption) features; $i i$ ) there is no clear signs of kinematics in molecular lines except for $\mathrm{CO}$ absorption lines at $\sim 2.4 \mu \mathrm{m}$.

\section{References}

Bik, A. \& Thi, W. F. 2004, AAp 427, L13

Blum, R. D., Barbosa, C. L., Damineli, A., Conti, P. S., \& Ridgway, S. 2004, ApJ 617, 1167

Bonnell, I. A., Bate, M. R., Clarke, C. J., \& Pringle, J. E. 2001, MNRAS 323, 785

Davies, B., Lumsden, S. L., Hoare, M. G., et al. 2010, MNRAS 402, 1504

Krumholz, M. R., McKee, C. F., \& Klein, R. I. 2005, ApJ 618, L33

Menezes, R. B., Steiner, J. E., \& Ricci, T. V. 2014, MNRAS 438, 2597

Murakawa, K., Lumsden, S. L., Oudmaijer, R. D., et al. 2013, MNRAS 436, 511

Navarete, F., Damineli, A., Barbosa, C. L., \& Blum, R. D. 2014, MNRAS, In preparation

Steiner, J. E., Menezes, R. B., Ricci, T. V., \& Oliveira, A. S. 2009, MNRAS 395, 64

Varricatt, W. P., Davis, C. J., Ramsay, S., \& Todd, S. P. 2010, MNRAS 404, 661 